\title{
Latest results on single electroweak boson production from ATLAS experiment
}

\section{Oleh Kivernyk, on behalf of the ATLAS Collaboration}

LAPP, Univ. Grenoble Alpes, Univ. Savoie Mont Blanc, CNRS/IN2P3, Annecy

E-mail: oleh.kivernyk@cern.ch

\begin{abstract}
We present the results on the latest measurements of single electroweak boson production performed with the ATLAS detector at the LHC. The new results are based on the 2012 and 20152016 data sets recorded at a center-of-mass energy of $\sqrt{s}=8 \mathrm{TeV}$ and $13 \mathrm{TeV}$, respectively. This document reports on a measurement of the triple-differential cross section for the Drell-Yan process $Z / \gamma^{\star} \rightarrow \ell^{+} \ell^{-}$in electron and muon decay channels and on measurements of the $W$ and $Z$ boson production cross sections in association with jets, including the measurement of electroweak $Z j j$ cross section. These measurements are sensitive to the parton distribution functions.
\end{abstract}

Sixth Annual Conference on Large Hadron Collider Physics (LHCP2018)

4-9 June 2018

Bologna, Italy 


\section{Introduction}

The measurements of the production of $W$ and $Z / \gamma^{\star}$ bosons at the LHC allow precise tests of most recent higher-order perturbative $\mathrm{QCD}(\mathrm{pQCD})$ and electroweak (EW) predictions. The large production cross sections and leptonic decay modes provide clean experimental signatures which can be precisely measured using ATLAS detector [1]. The predictions of vector boson cross sections in hadronic interactions depend on the parton distribution functions (PDF). Therefore, these measurements allow to test and to constrain models of parton dynamics at the LHC. The correct modeling of vector boson production is important in many physics analyses at the LHC for which they constitute an important background (Higgs boson measurements, new physics searches). Moreover, the measurement of triple-differential Drell-Yan cross section presented here is a crucial ingredient for a precise measurement of the effective weak mixing angle at the LHC.

\section{Triple-differential cross-section of Drell-Yan events at $8 \mathrm{TeV}$}

The presence of vector and axial-vector couplings of electroweak bosons to fermions in the Drell-Yan [2] processes $q \bar{q} \rightarrow Z / \gamma^{\star} \rightarrow \ell^{+} \ell^{-}$leads to a forward-backward asymmetry $A_{\mathrm{FB}}$ in polar angle $\theta$ between the lepton and incoming quark in the dilepton rest frame. This polar angle depends on the effective electroweak mixing angle, $\sin \theta_{\text {lept }}^{\text {eff }}$, whose precise knowledge provides a stringent test of the Standard Model. Previous extraction of the effective weak mixing angle in leptonic $Z$ boson decays from $A_{\mathrm{FB}}$ measurement has been performed by ATLAS using $7 \mathrm{TeV}$ data [3]. This result has the largest uncertainty due to limited knowledge of the PDFs. Here we report on the measurements of the triple-differential Drell-Yan cross section [8], $d^{3} \sigma /\left.d m_{\ell \ell} d\right|_{\ell \ell} \mid d \cos \theta^{\star}$, as a function of dilepton invariant mass $m_{\ell \ell}$, dilepton rapidity $\left|y_{\ell \ell}\right|$, and $\cos \theta^{\star}$, where the lepton decay angle is defined in the Collins-Soper (CS) reference frame [4]. These cross-section measurements are designed to be simultaneously sensitive to $\sin \theta_{\text {lept }}^{\text {eff }}$ and to the PDFs, giving the potential to reduce the PDF-induced uncertainty in the extracted value of the effective weak mixing angle.

The measurements of triple-differential cross sections are performed in the electron and muon decay channels for central rapidity region $\left|y_{\ell \ell}\right|<2.4$ and in the electron channel only for high rapidity region $1.2<\left|y_{\ell \ell}\right|<3.6$. The measured cross sections cover the kinematic range $46<$ $m_{\ell \ell}<200 \mathrm{GeV},\left|y_{\ell \ell}\right|<3.6$ and $-1<\cos \theta^{\star}<1$. The cross sections are classified as either forward $\left(\cos \theta^{\star}<0\right)$ or backward $\left(\cos \theta^{\star}>0\right)$ and are used to extract the $A_{\mathrm{FB}}$ in bins of $m_{\ell \ell}$ and $\left|y_{\ell \ell}\right|$ as:

$$
A_{\mathrm{FB}}=\frac{d^{3} \sigma\left(\cos \theta^{\star}>0\right)-d^{3} \sigma\left(\cos \theta^{\star}<0\right)}{d^{3} \sigma\left(\cos \theta^{\star}>0\right)+d^{3} \sigma\left(\cos \theta^{\star}<0\right)}
$$

The measurement consists of 504 bins for the central rapidity electron and muon channels, and 150 bins in the high rapidity electron channel. The bins are chosen as a compromise between the sensitivity to the underlying physics, the statistical precision, and the detector resolution effects. The contributions of electroweak and top backgrounds are estimated using MC simulation, whereas the contribution of events where a jet is faking a final state electron or muon is determined using a combination of data-driven methods and MC simulation. 


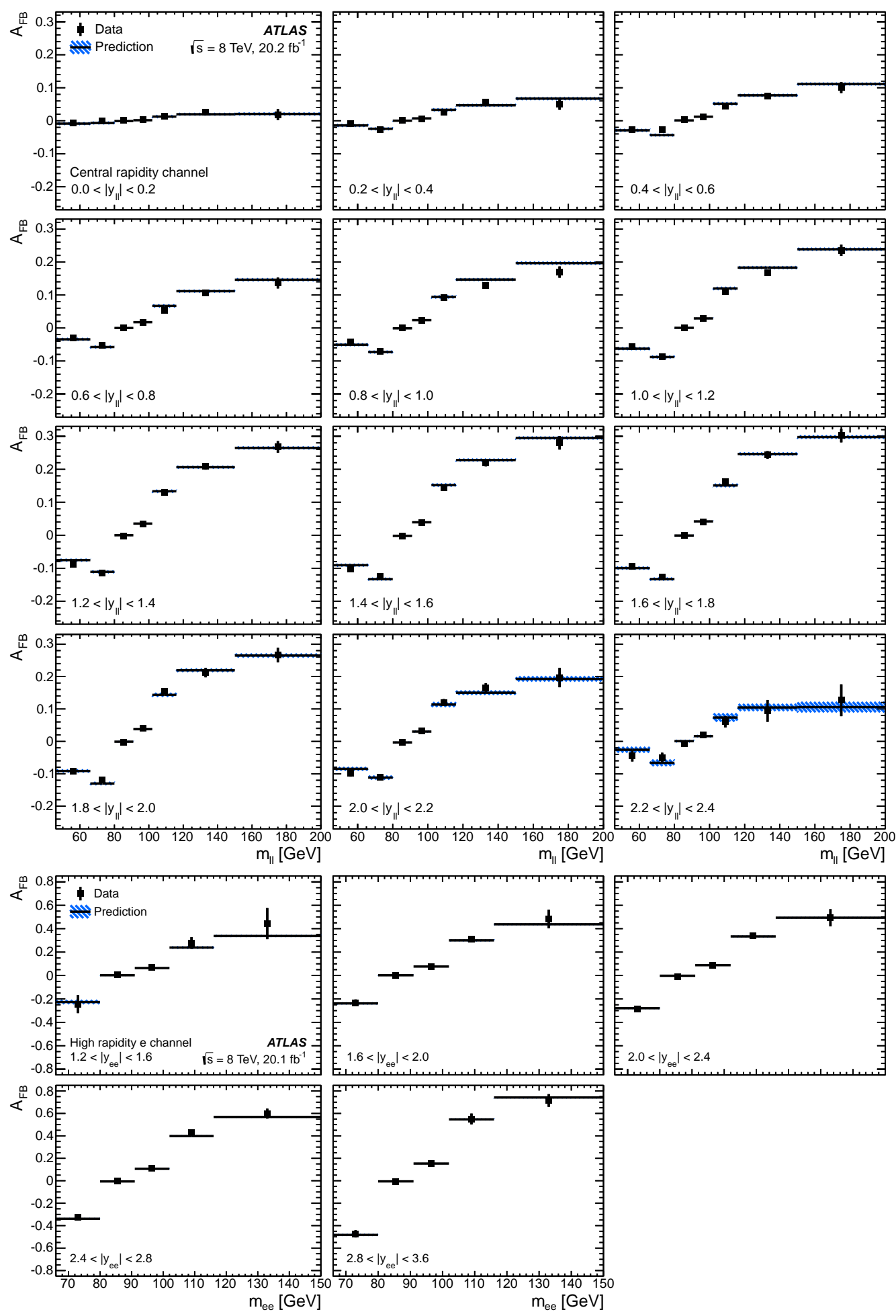

Figure 1: Forward-backward asymmetry, $A_{\mathrm{FB}}$, determined from the combined (top) and the high rapidity electron (bottom) Born-level fiducial cross section. The kinematic region is labelled in each plot. The data are shown as solid markers and the error bars represent the total experimental uncertainty. The prediction from POWHEG including NNLO QCD and NLO EW K-factors is shown as the solid line and the hatched band represents the statistical and PDF uncertainties in the prediction [8]. 
The central rapidity measurement channels are combined. Their combination achieves an experimental precision of better than $0.5 \%$, excluding the uncertainty in the luminosity measurement of $1.9 \%$. The statistical uncertainty is at the order of $0.5 \%$. The fiducial cross sections are compared to a theoretical prediction calculated using POWHEG+PYTHIA 8 with CT10 PDF set, including NNLO QCD and NLO EW $m_{\ell \ell}$-dependent K-factors. The measured cross sections were used to determine the $A_{\mathrm{FB}}$ as a function of $m_{\ell \ell}$ and $\left|y_{\ell \ell}\right|$. Figure 1 shows the measured $A_{\mathrm{FB}}$ compared to the predictions which are found to describe the observed behaviour well. These results can be used for a coherent determination of effective weak mixing angle and the PDFs.

\section{Vector boson production in association with jets.}

The study of vector boson production in association with jets at the LHC is of a great importance. The clean signatures in the leptonic decay channels make these events easy to identify and allow the vector boson kinematics to be well reconstructed. Such events are widely used for the calibration of the detector. Besides, $W / Z+$ jets events are backgrounds to many new physics searches. Therefore, it's extremely important to test the most advanced event generators that are used to model such events. This becomes possible with large samples of $p p$ data available from ATLAS. In the recent years, numerous theoretical predictions have become available including calculations for up to five additional jets at NLO and calculation for one additional jet at NNLO, as well as different merging approaches for NLO predictions, and various parton shower approaches.

\subsection{Z-boson production in association with jets at $13 \mathrm{TeV}$}

The measurement of $Z$-boson production in association with up to seven jets is reported here using $13 \mathrm{TeV}$ data recorded in 2015 with ATLAS [9]. The $Z$ boson is reconstructed using both electron and muon decay channels. The cross sections are measured as a function of inclusive and exclusive jet multiplicities and multiplicity ratios, the transverse momentum of the leading jet $p_{\mathrm{T}}^{\text {jet }}$ and its rapidity $y_{\text {jet }}$, the scalar sum $H_{\mathrm{T}}$ of the transverse momenta of all selected leptons and jets, and several other differential distributions involving jets. The jets are reconstructed with anti- $k_{\mathrm{T}}$ algorithm [5] within the radius of $R=0.4$. For this analysis, they are required to be with $p_{\mathrm{T}}^{\text {jet }}>30 \mathrm{GeV}$ and $\left|y_{\text {jet }}\right|<2.5$.

Figure 2 shows the comparison of measured cross sections with the various theoretical calculations for inclusive jet multiplicity and as a function of $H_{\mathrm{T}}$ for inclusive $Z+\geq 1$ jet events. The predictions of jet multiplicity are in agreement with the observed cross sections, except SHERPA 2.2, ALPGEN+Py6 and MG5_AMC+Py8 FXFX for high jet multiplicity where a non-negligible fraction of the jets is produced by the parton shower. The predictions from SHERPA 2.2, ALPGEN+PY6 and MG5_AMC+PY8 FXFX describe the $H_{\mathrm{T}}$ distribution well. The prediction from MG5_AMC+PY8 CKKWL overestimates the contribution at large values of $H_{\mathrm{T}}$. The fixed-order NLO prediction from BLACKHAT+SHERPA also underestimates the cross section at high $H_{\mathrm{T}}$ due to the missing contributions from events with higher parton multiplicities. But the agreement is recovered by adding higher orders in $\mathrm{pQCD}$, as demonstrated by the good description of $H_{\mathrm{T}}$ by $N_{\text {jetti }}$ NNLO [13]. 

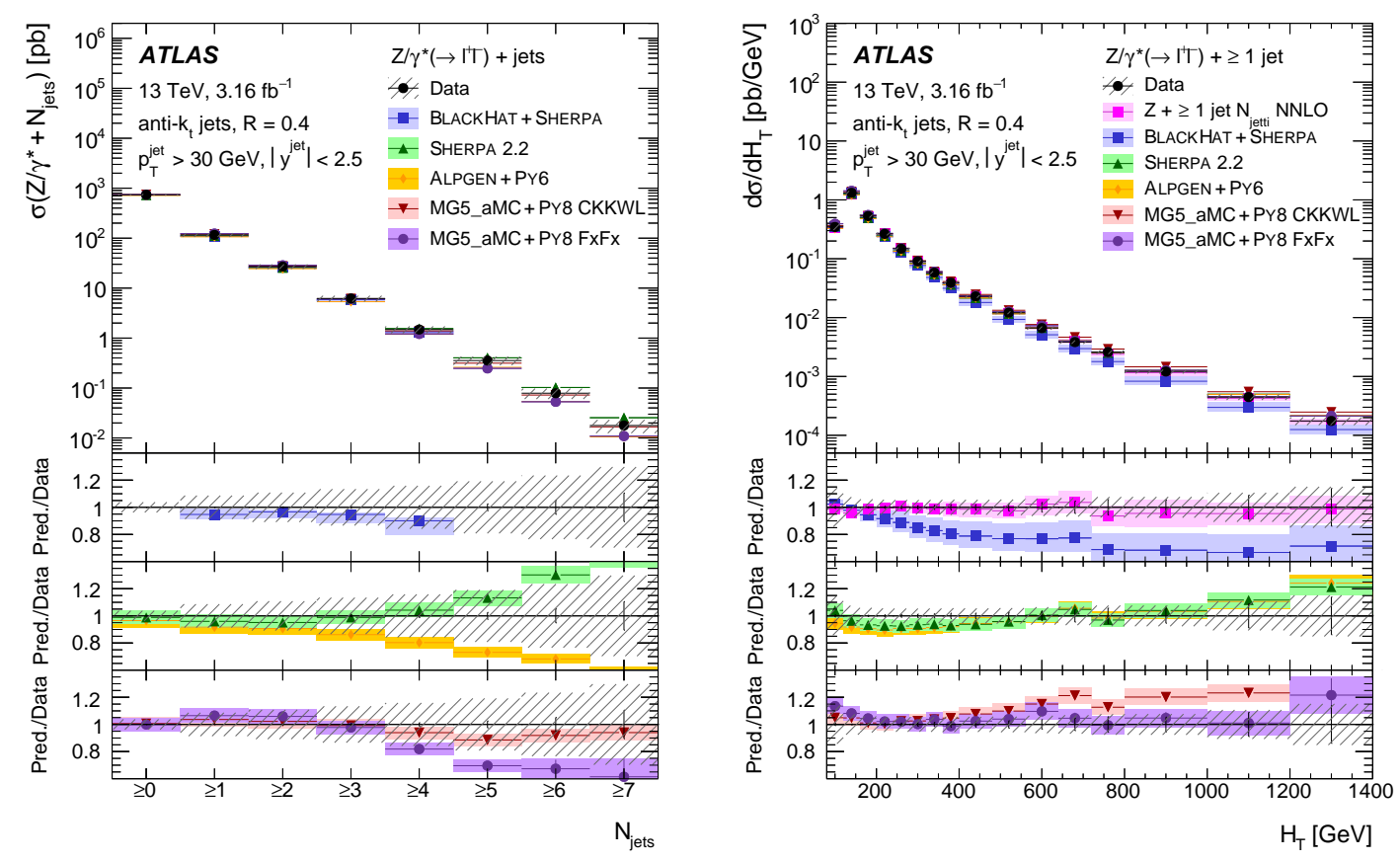

Figure 2: Measured cross section as a function of the inclusive jet multiplicity (left) and $H_{\mathrm{T}}$ (right) for $Z+$ jets events. The data are compared to the predictions from BLACKHAT+SHERPA, SHERPA 2.2, ALPGEN+Py6, MG5_AMC+PY8 CKKWL, and MG5_AMC+PY8 FXFX. The error bars correspond to the statistical uncertainty, and the hatched bands to the data statistical and systematic uncertainties (including luminosity) added in quadrature [9].

\subsection{W-boson production in association with jets at $8 \mathrm{TeV}$}

The measurement of $W$-boson production in association with up to seven jets is performed using $8 \mathrm{TeV}$ ATLAS data, recorded in 2012 [10]. The cross sections are measured as a function of jet multiplicity and eight kinematical observables which are reconstructed from jets and leptonic decay products of the $W$-boson, allowing to test $\mathrm{pQCD}$ under various conditions. The data are measured for $W, W^{+}, W^{-}$production and the cross section ratio $W^{+} / W^{-}$. Differential cross section measurements of $W^{+}$and $W^{-}$production and their ratio are sensitive to the PDFs for up and down quarks.

This measurement is performed in the electron decay channel $(W \rightarrow e v)$. The $W$ candidates are selected by requiring $p_{\mathrm{T}}^{e}>30 \mathrm{GeV}, E_{\mathrm{T}}^{\text {miss }}>30 \mathrm{GeV}$ and $m_{\mathrm{T}}^{W}>60 \mathrm{GeV}$, in order to reduce the backgrounds. The jets are reconstructed with anti- $k_{\mathrm{T}}$ algorithm [5] within the radius of $\mathrm{R}=0.4$. They are required to be with $p_{\mathrm{T}}^{\text {jet }}>30 \mathrm{GeV}$ and $\left|y_{\text {jet }}\right|<4.4$. To suppress the background from $t \bar{t}$ events, events with at least one b-tagged jet are rejected [6]. The electroweak and top backgrounds are estimated using MC, whereas the multijet background is determined using a data-driven method. The multijet background is the largest for events with less than four jets, whereas for five jets and above, $t \bar{t}$ dominates.

Figure 3 shows the comparison of measured cross sections with the various theoretical calculations for inclusive jet multiplicity and $H_{\mathrm{T}}$. Overall the data agree well for jet multiplicities. At higher multiplicities, the LO SHERPA predictions start to diverge from the data, while the NLO 
SHERPA predictions provide a much better description of the data. For $H_{\mathrm{T}}$, the LO predictions of SHERPA and ALPGEN, which both include multiple jets in the matrix element calculation describe the data best. The BLACKHAT+SHERPA predictions underestimate the data at large $H_{\mathrm{T}}$, due to additional jet emissions are missing. The exclusive sums approach in BLACKHAT+SHERPA achieves a similar agreement with data as NNLO. In both cases, the description of data is worse after inclusion of forward jets, comparing to $Z+$ jets in Figure 2.
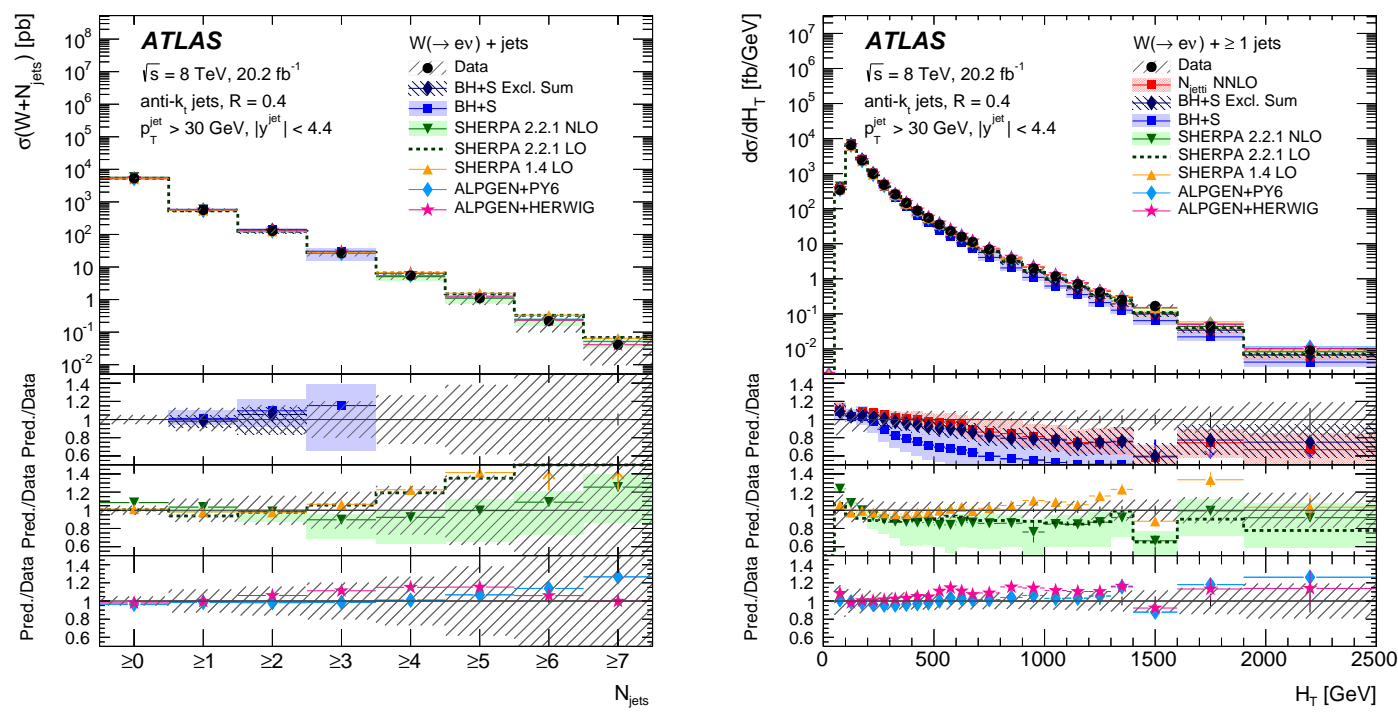

Figure 3: Measured cross section as a function of the inclusive jet multiplicity (left) and $H_{\mathrm{T}}$ (right) for $W+$ jets events [10].

\subsection{Electroweak production of dijets in association with a $Z$ boson at $13 \mathrm{TeV}$}

At the LHC, events containing a $Z$-boson and at least two jets $(Z j j)$ are produced via initialstate QCD radiation in Drell-Yan process (dominant QCD- $Z j j$ ) and via much rarer process of $t$-channel electroweak gauge boson exchange (EW- $Z j j$ ), including VBF. The kinematic properties of $Z j j$ events allow some discrimination between the QCD and EW production mechanisms. In particular, due to emission of virtual $W$-boson from the quark, two jets in EW production are harder and are more separated in terms of rapidity, leading to larger dijet mass. Moreover, EW-Zjj events are less likely to contain additional hadronic activity in the rapidity interval between two jets.

Here we report the results of measured fiducial cross sections for inclusive QCD+EW $Z j j$ and $\mathrm{EW} Z j j$ productions $13 \mathrm{TeV}$ data, recorded in 2015 [11]. The $Z$-boson is reconstructed using both the electron and muon decay channels, by requiring $p_{\mathrm{T}}^{\ell}>30 \mathrm{GeV}$ and $81<m_{\ell \ell}<101 \mathrm{GeV}$. Jets are reconstructed with anti- $k_{\mathrm{T}}$ algorithm and are required to have $p_{\mathrm{T}}^{j 1(j 2)}>55(45) \mathrm{GeV}$ and $|y|<4$.4. The measurements are performed in six phase spaces with different sensitivity to EW production. In addition to the baseline fiducial region, the QCD-enhanced region is defined by requiring a presence of at least one additional jet in the rapidity interval between two leading jets. In contrast, the two EW-enhanced regions are defined by applying jet veto in this interval and by requiring the dijet invariant mass to be $m_{j j}>250(1000) \mathrm{GeV}$. Other two regions are defined from 
baseline as the following: high- $p_{\mathrm{T}}$ region with increased cuts on $p_{\mathrm{T}}$ of jets $p_{\mathrm{T}}^{j 1(j 2)}>85(75) \mathrm{GeV}$, and high-mass region with $m_{j j}>1 \mathrm{TeV}$.

For the purpose of measurement of EW-Zjj cross section, the modeled QCD-Zjj component is corrected to data using QCD-enhanced region as shown in Figure 4 (left). For the QCD-Zjj simulations, three event generators are considered: ALPGEN, SHERPA and MG5_AMC. None of them do not describe $m_{j j}$ data well. In particular, SHERPA and MG5_AMC generators overestimate the data by $120 \%$ and $80 \%$ respectively, for $m_{j j}=2 \mathrm{TeV}$, well outside the modeling uncertainties.

Figure 4 (right) shows a summary of the fiducial cross-sections for this measurement compared to equivalent results at $8 \mathrm{TeV}$ and to theoretical predictions. As can be noticed, at higher dijet invariant masses $(>1 \mathrm{TeV})$, predictions from SHERPA (QCD-Zjj) + POWHEG $($ EW- $Z j j)$ and MG5_AMC (QCD-Zjj) + POWHEG (EW-Zjj) are found to significantly overestimate the observed $Z j j$ production rates in data. ALPGEN (QCD-Zjj) + PowHEg (EW-Zjj) provides a better description of the $m_{j j}$ shape.
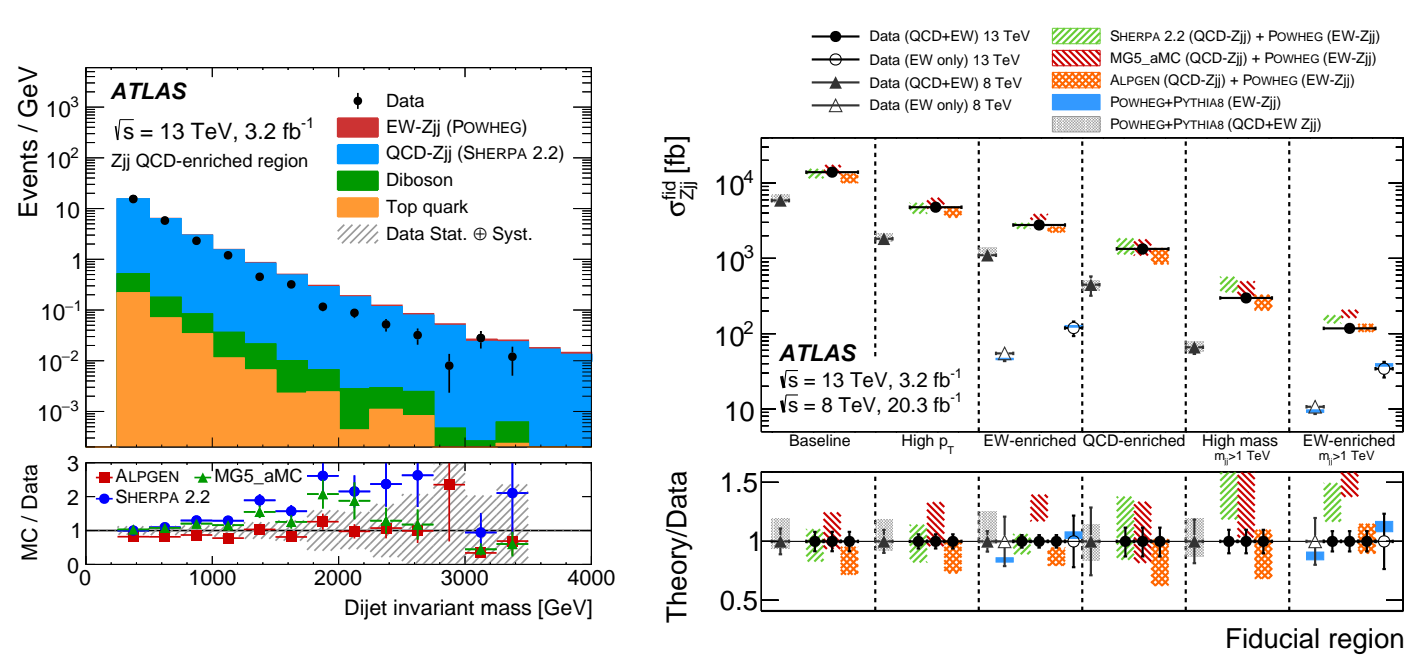

Figure 4: Left: detector-level comparisons of the dijet invariant mass distribution between data and simulation in the QCD-enriched region, for the dielectron and dimuon channel combined. Right: fiducial crosssections for a leptonically decaying $Z$ boson and at least two jets (solid data points) and $\mathrm{EW}-Z j j$ production (open data points) at $13 \mathrm{TeV}$ [11] (circles) compared to equivalent results at $8 \mathrm{TeV}$ [12].

\section{Summary}

This report summarizes the recent results of single vector boson studies using ATLAS detector at the LHC. These results include the measurements of triple-differential Drell-Yan crosssection, resulting into the measurement of forward-backward asymmetry $A_{\mathrm{FB}}$, that can be used for a coherent determination of effective weak mixing angle and the PDFs. We also report on the measurements of vector boson production cross sections in association with jets, including the measurement of EW-Z $j j$ cross section. These measurements provide a powerful test of most advanced event generators and of our understanding of pQCD. 


\section{References}

[1] ATLAS Collaboration, The ATLAS Experiment at the CERN Large Hadron Collider, JINST 3 (2008) S08003.

[2] S.D. Drell and T.-M. Yan, Massive Lepton-Pair Production in Hadron-Hadron Collisions at High Energies, Phys. Rev. Lett. 25 (1970) 316.

[3] ATLAS Collaboration, Measurement of the forward-backward asymmetry of electron and muon pair-production in pp collisions at $\sqrt{s}=7 \mathrm{TeV}$ with the ATLAS detector, JHEP 09 (2015) 049 [arXiv:1503.03709].

[4] J.C.Collins and D.E.Soper, Angular distribution of dileptons in high-energy hadron collisions, Phys. Rev. D 16 (1977) 2219.

[5] M. Cacciari, G. P. Salam and G. Soyez, The anti-kt jet clustering algorithm, JHEP 04 (2008) 063.

[6] ATLAS Collaboration, Performance of b-jet identification in the ATLAS experiment, JINST 11 (2016) P04008.

[7] ATLAS Collaboration, Measurement of the electroweak production of dijets in association with a $Z$-boson and distributions sensitive to vector boson fusion in proton-proton collisions at $\sqrt{s}=8 \mathrm{TeV}$ using the ATLAS detector, JHEP 04 (2014) 031.

[8] ATLAS Collaboration, Measurement of the Drell-Yan triple-differential cross section in pp collisions at $\sqrt{s}=8 \mathrm{TeV}$, JHEP 12 (2017) 059.

[9] ATLAS Collaboration, Measurements of the production cross section of a $Z$ boson in association with jets in pp collisions at $\sqrt{s}=13$ TeV with the ATLAS detector, Eur. Phys. J. C 77 (2017) 361.

[10] ATLAS Collaboration, Measurement of differential cross sections and $W^{+} / W^{-}$cross-section ratios for $W$ boson production in association with jets at $\sqrt{s}=8$ TeV with the ATLAS detector, JHEP 05 (2018) 077.

[11] ATLAS Collaboration, Measurement of the cross-section for electroweak production of dijets in association with a $Z$ boson in pp collisions at $\sqrt{s}=13 \mathrm{TeV}$ with the ATLAS detector, Phys. Lett. B 775 (2017) 206.

[12] ATLAS Collaboration, Measurement of the electroweak production of dijets in association with a $Z$-boson and distributions sensitive to vector boson fusion in proton-proton collisions at $\sqrt{s}=8 \mathrm{TeV}$ using the ATLAS detector, JHEP 04 (2014) 031.

[13] R. Boughezal, C. Focke, X. Liu and F. Petriello, W-boson production in association with a jet at next-to-next-to-leading order in perturbative QCD, Phys. Rev. Lett. 115 (2015) 062002. 